\title{
Network using Michaelis-Menten Kinetics: Constructing an Algorithm to Find Target Genes From Expression Data
}

\author{
Mythreye Krishnan, Michael Small, Anthony Bosco, Thomas Stemler
}

\begin{abstract}
The most challenging aspect of gene expression data analysis is to process the large and complex data using mathematical models and find biologically relevant information that gives insight to the underlying mechanism. We derived a simple ODE-based model using Michaelis-Menten Kinetics to process the microarray data. Different biological systems of experimental rhinovirus infection in humans, atopic CD4 T cell responses in allergens and responses to cancer immunotherapy in mice have been studied. The resulting analysis extracts highly linked target genes, the changes in which might cause changes in the other genes, in other words, potential targets for modulating gene network patterns and emergent biological phenotypes. We illustrate the application of the algorithm to identify novel targets in addition to previously identified targets in different experimental contexts.

Keywords: Complex Networks, Gene expression data, Michaelis-Menton Kinetics, Mathematical modelling

Classification: Complex Networks, Systems Biology
\end{abstract}

\section{Introduction}

\subsection{Complex Networks}

Gene Regulatory Networks (GRN) study the effect of one gene on another and form a network of gene interactions. For example, gene A can regulate gene B by either enhancing the activity or suppressing the expression and activity of gene B. Gene A can also enhance or suppress its own expression via a feedback loop. Genomic data is obtained using microarray analysis. Complementary strands of DNA are either radio-labelled or labelled with fluorescent markers and affixed to a chip. The position of the affixed strands are indexed for identification. The mRNA, expression of which is to be measured, is then allowed to bind to the chip and the amount of binding is measured as colour intensities. The colour intensities are then converted into a series of numbers which are stored in the form of a table or matrix. Building a GRN from genomic data is the most challenging aspect as it needs to identify the biological correlation [Lee et al., 2012] using models instead of experiments. Reverse engineering allows us to work backwards form experimental data to infer the underlying network structure [Sacchi et al., 2007, Wang et al., 2014]. The data can be a time-series experiment or biological response to a perturbation. Time course data is recording the expression levels of the genes at regular intervals and perturbed data contains varying gene expressions following genetic perturbation (gene silencing or over-expression), expression under different environmental conditions for example, in response to a stimulus. The main step in processing microarray data to form networks is the analysis of the data. Even though the data obtained from microarray experiments are large, the actual process itself is very expensive [Alvarez et al., 2016]. In order to reduce the experimental dependence of validating each gene, in the data analysis step, a mathematical function is used to analyze the large volume of data [Janes et al., 2006] and deduce the correlation between the genes to build a network of relations to form a model architecture [Hecker et al., 2009]. The resulting network is based on the gene expression and the type of correlation applied on the data. Distinguishing the direct interactions and the correlations [Feizi et al., 2013] will give us a reduced number of targets which can then be further analyzed in a cost-effective manner. Mathematical methods like Principal Component Analysis (PCA) compresses the data and analyzes it to find useful patterns [Abdi et al., 2010, Kossenkov et al., 2010] and also can be used to cluster the genes using their expression values [Kim et al., 2011]. Integration of results from different approaches have been shown to improve the computational analysis of GRN [Chan et al., 2008]. Our aim is to use a novel dynamical method that can build a network of genes with focus on the high betweenness centrality nodes that has biologically relevant information instead of finding patterns using just numerical values by analyzing the change in expression of all genes on one another individually, including the gene on itself by assuming a feedback loop.

The dynamics of biological systems come from the gene interactions in the cell [Képés et al., 2007]. The 
basic problem in forming network structure from genomic data is identifying the corresponding kinetic parameters and inferring the underlying dynamics [Ronen et al., 2002]. Stochastic modeling in biological systems allows us to incorporate randomness [Kulkarni et al., 2016, ?] there-by enabling the data processed to be either discrete or continuous. Some of the mathematical approaches that are commonly applied for studying the dynamics of the networks are Boolean, Bayesian and Differential equations depending upon the biological level to be studied from abstract to concrete biological network. Abstract methods can incorporate large volume of data to identify qualitative links with less biological relevance while concrete dynamics like stochastic modeling are closer to biological reality [Lee et al., 2009]. A similarity matrix derived from the data processing is used to identify the links between the genes to build networks. Networks are weighted with values corresponding to the edges and the edge weight typically dictates the strength of the connectivity [Feizi et al., 2013] between the genes. Network based approaches are useful in finding genes and pathways that are associated in diseases and can aid in better target-oriented drug development [Barabási et al., 2011].

Boolean Networks are the simplest existing method to find the links between the genes. Each gene in the network is either activated/expressed or suppressed. They are turned on or off conditionally and the expressions of the genes under different conditions is used to study the underlying dynamics of the system [Wang et al., 2013]. The resulting binary network shows that each gene is influenced by one or more genes and edges are defined by the connectivity obtained by thresholding and/or clustering [Hecker et al., 2009, Képés et al., 2007]. The limitations of this method are the increasing complexity with the increasing amount of data [Lee et al., 2009] and the gene expression models cannot be described with simple on and off functions [Hecker et al., 2009] and also they fail to capture a lot of underlying dynamics.

Bayesian Network analysis generates a directed acyclic graph (DAG) that establishes a probabilistic relation between the network nodes [Lee et al., 2009]. They are useful in finding a set of closely interacting components as each component is dependent upon the value of few components [Friedman et al., 2000]. There are two steps in establishing dynamics using Bayesian networks: first is model selection, finding the DAG and next is parameter learning, estimating the probabilities that govern the relation between the genes [Lee et al., 2009, Zou et al., 2004]. The model selection is more crucial as it governs the relation between the nodes and hence few DAGs are chosen for the same dataset and scored to find the best fitting model [Hecker et al., 2009]. This method strongly driven by the chosen priors [Moloshok et al., 2002] and is more suitable to analyze when one expression of one gene is influenced by few other genes and is helpful in finding local structures [Friedman et al., 2000].

When using differential equations regulation of a gene is expressed as the rate of change in the expression of mRNA influenced by other genes [Képés et al., 2007, Hecker et al., 2009]. Simple ordinary differential equations ODE that are non-linear differential equations can describe the dynamics of the system more accurately than linear ODE [Lee et al., 2009] but can only search on a smaller sample size in comparison to the other methods for structure identification [Hecker et al., 2009]. Using differential equations to study the dynamics is computationally very expensive and can handle only datasets of limited size.

We propose a novel algorithm to study the dynamics using a non-linear differential equation derived from Michaelis-Menten kinetics (MMK). This method gives an advantage of the results not being influenced by the prior knowledge and help to test new hypotheses and thus, identify novel patterns and targets [Kong et al., 2011]. We assume that each gene contributes to some change in every other gene in the system by applying the algorithm to the expression data. Using the dynamics, we obtain a large similarity matrix. An adjacency matrix is built by applying a threshold to the similarity matrix and betweenness centrality is calculated for the adjacency matrix to find the target nodes. The number of target genes varies with the threshold used; the threshold is selected by applying node entropy and is described in section 3.2. The higher the threshold, lower the target nodes and vice versa. The target genes obtained are evaluated for prior association in the literature and novel gene nodes are proposed as potential experimental targets. 


\section{Approach}

The aim of our study is to find the highly connected target nodes the deletion of which, is predicted to bring maximum change to the other genes. Each gene is considered as a node and for a given data matrix $\mathbf{D}_{\mathbf{m} \times \mathbf{n}}$, there are $m$ genes from $n$ observations. We apply a novel method to find the highly connected nodes based on the actual expression of the genes. We do a pairwise comparison of the gene expression from the microarray data and obtain the kinetic parameters for each pair of genes. One of the requirements for the application of this model is that the gene has to be expressed in at least two different states and we compute the difference between the states. We observe how each gene expression changes under different conditions and use it as a driving factor to find the actual similarity. Some of the other assumptions made for this model are that we do not study the effect of more than one gene and each gene is assumed to have a basal level of expression and is explained in section 3.1.

\section{Method}

\subsection{Algorithm}

The rate of change of gene expression with respect to other genes is calculated using the Michaelis-Menten Kinetics (MMK) as described in Appendix A.

We first calculate the expression change $\mathbf{G}$, by finding the difference between the control and the study of interest for each observation; for example, the control and the infected state for rhinovirus dataset are paired. Each pair of observation comes from the same individual before and after exposure to rhinovirus. If the samples are not paired, we take the mean value of the control conditions for each gene; in cancer immunotherapy dataset, we have mice that responsive and non-responsive to immunotherapy. We calculate $\mathbf{G}$ as follows. An element $D_{i j}$ of the dataset $\mathbf{D}_{\mathbf{m} \times \mathbf{n}}$ represents the expression of $i^{\text {th }}$ gene for $j^{\text {th }}$ observation. Let $\mathbf{e}_{\mathbf{i}}$ denote the vector that is the $i^{\text {th }}$ row of $\mathbf{D}_{\mathbf{m} \times \mathbf{n}}(\mathbf{m} \gg \mathbf{n})$. This vector $\mathbf{e}_{\mathbf{i}} \in \mathbf{R}^{\mathbf{v}}$ contains $\mathbf{c}_{\mathbf{i}}$ controls and $\mathbf{s}_{\mathbf{i}}$ samples, such that $\mathbf{e}_{\mathbf{i}}=\left(\mathbf{c}_{\mathbf{i}}, \mathbf{s}_{\mathbf{i}}\right)$. Both $\mathbf{c}_{\mathbf{i}}$ and $\mathbf{s}_{\mathbf{i}}$ are constant $\forall i$ in a given dataset. If $\mathbf{c}_{\mathbf{i}}$ and $\mathbf{s}_{\mathbf{i}}$ are the same length, the change in expression $\left(\mathbf{g}_{\mathbf{i}}\right)$ for each gene vector is calculated by finding element-wise difference.

$$
\mathrm{g}_{\mathrm{i}}=\mathrm{s}_{\mathbf{i}} \mathbf{c}_{\mathbf{i}}
$$

If $\mathbf{c}_{\mathbf{i}}$ and $\mathbf{s}_{\mathbf{i}}$ are of different lengths, we find mean of control vector for each gene and then find the difference of that scalar value from the samples.

$$
\mathbf{g}_{\mathbf{i}}=\mathbf{s}_{\mathbf{i}}\left(\overline{\mathbf{c}_{\mathbf{i}}} \cdot \mathbb{1}\right)
$$

Let $r$ be the length of the vector $\mathbf{s}_{\mathbf{i}}$. As we find the difference between the sample vector and a scalar, we multiply the mean control $\left(\mathbf{c}_{\mathbf{i}}\right)$ with $\mathbb{1}$, an $r$ dimensional vector of all ones, to perform element-wise calculation. By finding the difference for each gene, we obtain a matrix $\mathbf{G}$ of size $m \times r$ ( $r$ is the number of samples). We then calculate the kinetic parameters and the similarity value between each pair of genes in $\mathbf{G}$ by applying equation given in 2 .

Our model has three basic terms. A basal level expression of the gene (it is based on the assumption that any gene will always be present in a minimum amount in the cell at all times), degradation of the gene and the change in expression of the gene, governed by Michaelis-Menten Kinetics mentioned in Appendix A. Any row vector $\mathbf{g}$ in $\mathbf{G}$ has $r$ elements. The similarity between any two vectors in the matrix $\mathbf{G}, \mathbf{g}_{\mathbf{i}}$ and $\mathbf{g}_{\mathbf{j}}$ (where $i, j=1, \ldots, m$ and $m$ is the total number of genes) is calculated as follows.

Let $B$ be the basal level of gene expression and is calculated as,

$$
B=\frac{\min \left(\mathbf{g}_{\mathbf{i}}\right)}{n}
$$

where $\min \left(\mathbf{g}_{\mathbf{i}}\right)$ is the smallest element of vector $\mathbf{g}_{\mathbf{i}}$ and $n$ is the total number of observations. This equation is calculated based on the assumption that there will always be a basal level expression of a gene present in a cell. Any change in the gene occurs from this value.

$$
K_{d}=B \cdot \frac{\min \left(\mathbf{g}_{\mathbf{i}}\right)}{2}
$$


When a gene reaches a certain concentration in the cell, it gets degraded. $K_{d}$ is calculated from the values of the vector $\mathbf{g}_{\mathbf{i}}$.

The Michaelis-Menten equation for the rate of product formation is given by (Refer Appendix A),

$$
v=\frac{V_{m} \times[S]}{K_{m}+[S]}
$$

Let $V$ (the value at which the change in $g_{i}$ is maximum) and $K$ (the value at which the change in $g_{i}$ is at half-maximum) correspond to the kinetic parameters $V_{m}$ and $K_{m}$ of the Michaelis-Menten equation. By rearranging the equations, in equilibrium, the rate of the reactions are dependent on the concentrations of the substrate, enzyme and product (See Appendix A). So, we derive the values of $K_{d}, K$ and $V$ to be dependent on the expression of $g_{i}$ and $g_{j}$. The gene expression vectors $\mathbf{g}_{\mathbf{i}}$ and $\mathbf{g}_{\mathbf{j}}$ are dimensionless at gene expression level. Hence, all other quantities including $B, K_{d}, K$ and $V$ are also dimensionless. For our algorithm, the values are calculated by,

$$
K=\frac{\max \left(\mathbf{g}_{\mathbf{i}}\right)}{B \cdot \min \left(\mathbf{g}_{\mathbf{i}}\right)}
$$

where, $\max \left(\mathbf{g}_{\mathbf{i}}\right)$ is the largest element of vector $\mathbf{g}_{\mathbf{i}} . K$ is a value substituted for the rate constant $K_{m}$ in 1 at which the velocity of change in $\mathbf{g}_{\mathbf{i}}$ is at half-maximum.

$$
V=\max \left(\mathbf{g}_{\mathbf{i}}\right) \cdot \overline{\mathbf{g}_{\mathbf{j}}}
$$

where, $\overline{\mathbf{g}_{\mathbf{j}}}$ is the mean of all change in expression for gene $j$. The term for relative change in gene $i$ with respect to gene $j$ governed by Michaelis-Menten Kinetics is given by,

$$
\frac{V \cdot \overline{\mathbf{g}_{\mathbf{i}}}}{K+\overline{\mathbf{g}_{\mathbf{i}}}}
$$

Thus, $\left\{B, K, K_{d}\right\}=f\left(g_{i}\right)$ and $V=f\left(g_{i}, g_{j}\right) . V$ correspond to $V_{m}=k_{2} \times E_{0}$ (Appendix A), the rate at which the velocity is maximum; it is dependent on the initial enzyme concentration in Michaelis-Menten Kinetics. Here, $V$ is the point at which the change in gene $i$ is completely saturated by gene $j$ catalysing the change in gene $i$ and is dependent on the concentration or change in the levels of gene $j$. The values of $V$ (See Appendix A, $V$ is dependent on the initial enzyme concentration, $V_{m}=k_{2} \times E_{0}$ ) and $K$ (See Appendix A, $K$ is the ratio of the rates $k_{-1}$ and $k_{1}$, the rates from reversible binding of substrate with enzyme) are derived directly from the values of the vectors $\mathbf{g}_{\mathbf{i}}$ and $\mathbf{g}_{\mathbf{j}}$. We do not take into consideration the intermediary factors, like transcription factors or binding of $\mathbf{g}_{\mathbf{j}}$ or other proteins that could influence this change.

The change in $\mathbf{g}_{\mathbf{i}}$ is influenced by the change in $\mathbf{g}_{\mathbf{j}}$. As we apply the Michaelis-Menten kinetics, we study the effect of $\mathbf{g}_{\mathbf{j}}$ acting like an enzyme to alter the expression of $\mathbf{g}_{\mathbf{i}}$. The model equation to calculate the similarity $p_{i j}$ between each pair of genes is obtained by combining the three terms and is given by,

$$
p_{i j}=B-K_{d} \cdot \overline{\mathbf{g}_{\mathbf{i}}}+\frac{V \cdot \overline{\mathbf{g}_{\mathbf{i}}}}{K+\overline{\mathbf{g}_{\mathbf{i}}}}
$$

The similarity measure is a function of both genes $i$ and $j, p_{i j}=f\left(g_{i}, g_{j}\right)$. The vector $\mathbf{p}_{\mathbf{i}}(i=1, \ldots, m)$ represents the relative change in expression of a gene $g_{i}$ at any point with respect to the other genes, $\mathbf{g}_{\mathbf{j}}$.

This step is repeated across the entire dataset by performing a pairwise comparison of all the genes, including the gene on itself. Thus, we obtain a similarity matrix $\mathbf{P}$. Varying the kinetic parameters by applying minimum, maximum or mean values interchangeably have little or no significance on the results; using the individual values like time points is beyond the scope of this manuscript and could be a path for potential future exploration.

When we derive the matrix $\mathbf{G}$ some of the values are negative; this is due to the fact that when we find the difference between the control and the study of interest, there are some genes that get suppressed and their value decreases with respect to control. So, some values of $K$ become negative and the 
equation has to be modified to incorporate the substrate inhibition. If the gene is inhibited, then the equation is modified to,

$$
p_{i j}=B-K_{d} \cdot \overline{\mathbf{g}_{\mathbf{i}}}+\frac{V \cdot \overline{\mathbf{g}_{\mathbf{i}}}}{K+\left(2 \cdot \overline{\mathbf{g}_{\mathbf{i}}}\right)}
$$

This equation is used when the gene $i$ inhibits any other gene $j$ competitively, and is not altered by change in gene $j$. As Michaelis-Menten Kinetics cannot have a negative value for the rate, $K$ and in order to adhere to the rules of the kinetics, we assume that the gene is competitively inhibited by itself, as we study the effect of only one gene at a given time. So the equation is modified by substituting the value of $\left(K+\overline{\mathbf{g}_{\mathbf{i}}}\right)$ with $\left(K+\left(2 \cdot \overline{\mathbf{g}_{\mathbf{i}}}\right)\right)^{1}$.

Processing all the genes through the equation gives us a large similarity matrix $\mathbf{P}$ of size $m \times m$, where $m$ is the number of genes. For an absolute maximum value of $p_{i j}$, the gene $g_{j}$ maximizes the gene $g_{i}$. Irrespective of whether the value of $p_{i j}$ is positive or negative, the gene $g_{j}$ is assumed to either enhance or suppress the effect of gene $g_{i}$. We combine the similarity matrix $\mathbf{P}$ and its transpose $\mathbf{P}^{\mathbf{T}}$ to obtain a symmetric matrix; as the equation 2 is non-linear and we need to build a symmetric adjacency matrix from the similarity matrix to find the highly connected nodes, we calculate the summation.

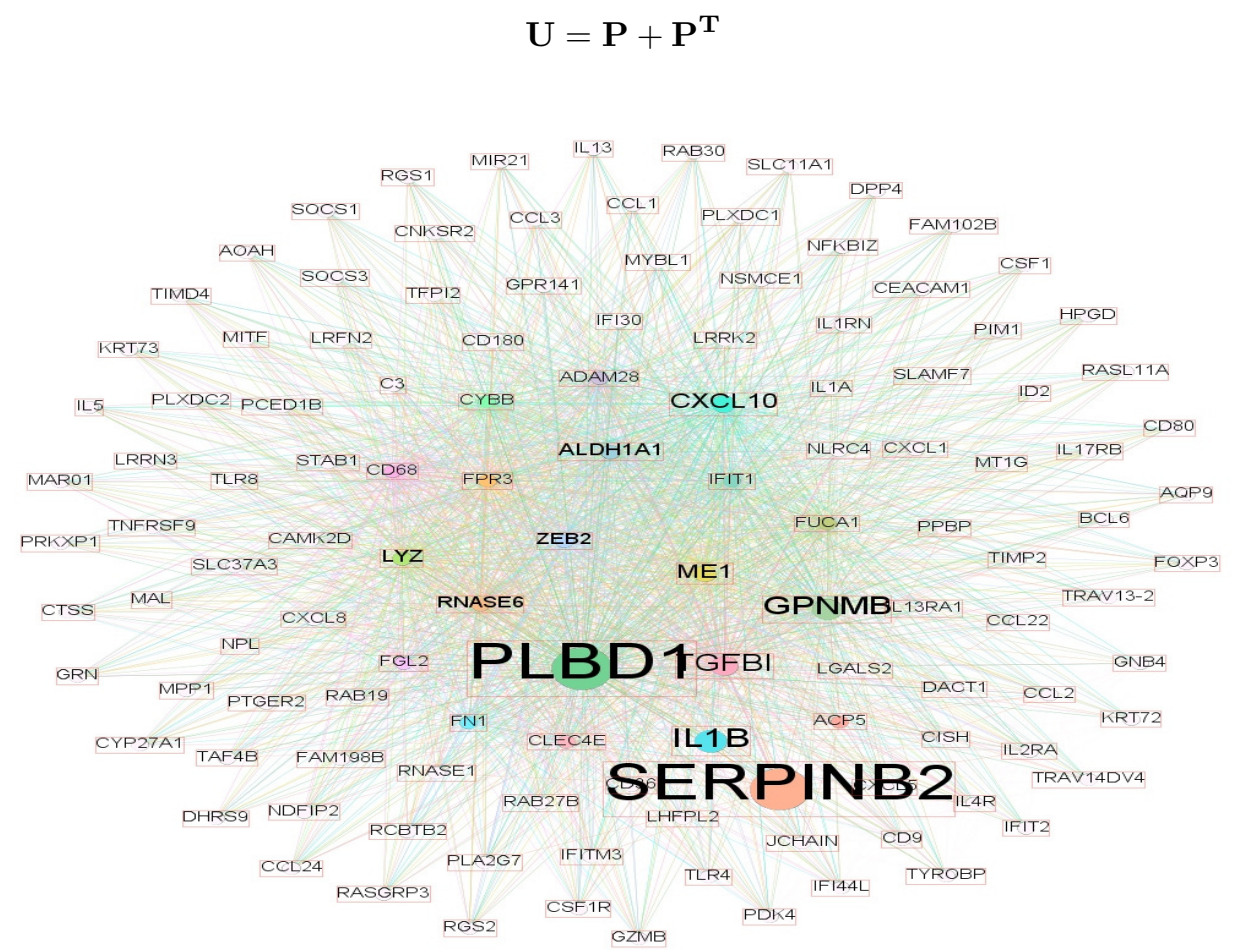

Figure 1: Network representation of individual genes are represented with their corresponding gene names. This is a compact representation of the application of network algorithm for the allergy dataset highlighting the high betweenness centrality nodes. The size of the node and gene name is proportional to their respective betweenness centrality values (PLBD1, SERPINB2, IL1 $\beta$, TGFBI, GPNMB, CXCL10, ALDH1A1, ME1, LYZ, RNASE6).

\subsection{Node Entropy}

For a given adjacency matrix $\mathbf{A}$, with an element denoted by $a_{i j}$, the node entropy of node $i$ for an unweighted matrix is calculated as,

$$
\mu(i)=-\sum_{j, a_{i j} \neq 0} p(i, j) \log p(i, j)
$$

\footnotetext{
${ }^{1}$ http://www1.lsbu.ac.uk/water/enztech/inhibition.html
} 


$$
p(i, j)=\frac{a_{i j}}{\sum_{k} a_{i k}}
$$

\subsection{Building an Adjacency Matrix}

The adjacency matrix $\mathbf{A}$ is calculated from the matrix $\mathbf{U}$ by applying a threshold. In the absence of a threshold, we get a completely connected graph with varying degree of connectivites. Our focus is to obtain nodes that could be used as potential experimental targets and not on the network properties or structure. The optimum threshold to build an adjacency matrix is chosen by applying node entropy to a range of thresholds. We identify the point at which there is a change in the node entropy and apply that transition point to obtain an optimum number of high betweenness centrality nodes. We obtained 20 to 50 high betweenness centrality nodes for the three datasets of study. The number of high betweenness nodes varies due to various factors like the size of the dataset and the similarity values. We used an unweighted adjacency matrix for further calculations, by applying betweenness centrality.

\subsection{Betweenness Centrality}

Betweenness centrality of a node is a measure of the extent to which this node can exert control over other nodes in the network [1, 2, White et al., 1994]. Since this method works only for networks that do not contain multiple edges [2], we apply this to our adjacency matrix to find the nodes that control other nodes. Betweenness centrality is a measure of ratio of $u_{a b}$ the sum of number of times a node $u$ occurs in the shortest paths between nodes $a$ and $b$ and $N_{a b}$, the total number of shortest paths between nodes $a$ and $b$.

$$
B C=\sum_{a, b} \frac{u_{a b}}{N_{a b}}
$$

The disruption or change in these nodes are likely to bring a lot of change in the other nodes that are present in the system [White et al., 1994].

\subsection{Testing the Approach}

As we have developed a novel approach to analyse the gene expression data from microarray expression, we have tested it on few open source datasets from Gene Expression Omnibus (GEO). The first one, breast cancer dataset with tumors from Estrogen Receptor Positive $(\mathrm{ER}+)$ and tumors from patients with Estrogen Receptor negative (ER-), GEO ID GDS4085. There are 20 samples in total, 9 ER + tumor samples and 11 ER- tumor samples with 1218 genes. The second one is Yeast grown in the presence and absence of Hydrogen Peroxide $\mathrm{H}_{2} \mathrm{O}_{2}$ for Superoxide dismutase deficient gene (SOD) with GEO ID GDS5630. As we applied the approach, we found that the algorithm identified the genes that relevant to study of interest. By applying the node entropy, the ER+ Control had 19 high betweenness centrality nodes, ER- had 30 and Yeast had 6 nodes.

Table 1: GEO datasets-Breast Cancer and Yeast

\begin{tabular}{|c|l|l|l|l|l|l|l|}
\hline No. Dataset & SampleState1 & $\begin{array}{l}\text { No. } \\
\text { of } \\
\text { Samples }\end{array}$ & State2 & $\begin{array}{l}\text { No. } \\
\text { of } \\
\text { SamplesGenes }\end{array}$ & $\begin{array}{l}\text { No. } \\
\text { of }\end{array}$ \\
\hline 1 & $\begin{array}{l}\text { Breast } \\
\text { Cancer } \\
\text { Yeast }\end{array}$ & 22 & $\begin{array}{l}\text { ER }+ \\
\text { Tumor } \\
\text { SOD1 } \\
\text { Deficient } \\
\text { in pres- } \\
\text { ence } \\
\text { of } \mathrm{H}_{2} \mathrm{O}_{2}\end{array}$ & 9 & $\begin{array}{l}\text { ER- } \\
\text { Tumor } \\
\text { SOD1 } \\
\text { deficient } \\
\text { in ab- } \\
\text { sence } \\
\text { of } \mathrm{H}_{2} \mathrm{O}_{2}\end{array}$ & 2 & 11218 \\
\hline
\end{tabular}

For breast cancer data, it was curated with reduced number of genes and did not have controls. So, we found the difference between the two states interchangeably and applying the algorithm identified 
CXCL14, GFRA1 and ERBB4 as top ranked nodes when ER+ was used as control. Similarly, CXCL9, CXCL13 and CX3CL1 were top ranked for ER- as control. As we analyse only the high betweenness centrality nodes, we verified the known associations for the top nodes in the literature. As described by Palacios et al., [Palacios-Arreola et al., 2014], the chemokines and their receptors (CXCL9, CXCL14, CX3CL1) have significant impact in breast cancer tumors. Similarly, CXCL13 is over-expressed in breast cancer tumors and is proven by Panse et al [Panse et!al., 2008] and ERBB4 shows significant increase in expression for ER+ breast cancer tumors [Junttila et al., 2005]. GFRA1 is up-regulated in ER+ breast cancer tumors and has already been studied to be a potential experimental target [Bhakta et al., 2018]. All the top high betweenness centrality genes have known associations with breast cancer, as the dataset is curated to contain only genes that are relevant to the study of interest.

Similarly, for Yeast dataset, we obtained SOD1, GOR1 and HBT1; the Superoxide dismutase gene (SOD1) that enables the organism to grow when subjected to environmental stress with $\mathrm{H}_{2} \mathrm{O}_{2}$ [Montllor et al., 2019] and is the study of interest for this dataset; it had the highest betweenness centrality value. GOR1 has an oxido-reductase activity and increases in DNA replication stress ${ }^{2}$.HBT1 is involved in the stationary phase of budding and a novel gene for the study of interest [Dittmar et al., 2002].

ERBB4 and GFRA1 are over-expressed and up-regulated in ER+ breast cancer tumors. SOD1 had the highest betweenness centrality value and the largest hub we obtained for yeast dataset that has more than 10000 genes. Encouraged by the results, we proceeded to test the algorithm with other datasets to verify if the method can identify novel targets for further studies.

\subsection{Data}

The rhinovirus dataset comprises microarray data derived from nasal scrapings collected before and after experimental rhinovirus infections. Study was conducted in vivo in randomized individuals who were infected with Human Rhino virus (HRV), HRV-16 and another group, which was sham inoculated. The samples have been collected 14 days before inoculation and twice at 8 hours and 48 hours post-inoculation. Samples collected after 8 hours did not have significant changes with respect to sham inoculation while the samples collected at 48 hours showed significant changes in the chemokine and interferon-inducible genes [Proud et al., 2008].

The allergy dataset has samples from three different clinical groups of subjects. House dust mites (HDM) sensitized atopics with current asthma, HDM sensitized atopics without current asthma and HDM nonsensitized controls. Peripheral blood mono-nuclear cells (PBMC) was cultured in the presence or absence of HDM for 24 hours; CD4 T cells were isolated employing immunomagnetic separation at the termination of the cultures, total RNA was extracted and subjected to microarray analysis [Troy et al., 2016].

For cancer immunotherapy data, immune checkpoint blockade therapy with antibodies that target $\mathrm{Cy}$ totoxic T-lymphocyte antigen (CTLA) 4 was studied in a murine mesothelioma model. This model is characterised by a dichotomous response to the therapy. Mice that were in identical environment were given anti-CTLA4; 7 days after the treatment the tumor was removed and the regressor and progressor tumors were studied using microarray expression. The study was also performed to obtain data from re-purposed drugs that can potentially be used in combination with anti-CTLA4 [Lesterhuis et al., 2015].

\subsection{Ingenuity Pathway Analysis}

Ingenuity pathway analysis (IPA) is a software application hosted by Qiagen and a repository of life science information; it is web-hosted and can be used for biological and chemical analyses. Given a gene list, IPA can be used to identify the molecular mechanisms, the pathways and processes associated with them. Upstream Regulatory Analysis (URA) is a part of IPA, where, the software can produce a set of upstream regulators that can inhibit or activate a given set of genes. The regulators are ranked according to the URA by placing the most significant upstream regulator on the top ${ }^{3}$. We use IPA-URA to validate the algorithm by verifying if the high betweenness centrality nodes identified by the algorithm are upstream regulators of the nodes that are linked to them. The top 10 high betweenness centrality

\footnotetext{
${ }^{2}$ https://www yeastgenome.org/locus/S000005218

${ }^{3}$ The regulators are ranked based on the overlap p-value calculated using Fisher's Exact Test, and significance is generally attributed to $p<0.01[3]$
} 
Table 2: Sample size of each dataset. The rhinovirus dataset has PBMC samples with control and rhinovirus infected states. Allergy dataset has patients having onset of asthma after exposure to dustmites and healthy controls. Cancer immunotherapy has mice that are responsive to the treatment study and mice that are not responsive.

\begin{tabular}{|c|c|c|c|c|c|c|c|}
\hline No. & Dataset & \multicolumn{2}{|c|}{$\begin{array}{l}\text { SampleState1 } \\
\text { Size }\end{array}$} & $\begin{array}{l}\text { No. } \\
\text { of } \\
\text { Sam }\end{array}$ & State2 & $\begin{array}{l}\text { No } \\
\text { of } \\
\text { Sar }\end{array}$ & $\begin{array}{l}\text { No. } \\
\text { of } \\
\text { sGenes }\end{array}$ \\
\hline 1 & Rhinovirus & 30 & Control & 15 & $\begin{array}{l}\text { Infected with } \\
\text { Rhinovirus }\end{array}$ & 15 & 5367 \\
\hline 2 & Allergy & 44 & Control & 22 & $\begin{array}{l}\text { HDM } \\
\text { stimulation }\end{array}$ & 22 & 2564 \\
\hline 3 & $\begin{array}{l}\text { Cancer } \\
\text { Immuno- } \\
\text { therapy }\end{array}$ & 20 & $\begin{array}{l}\text { Non- } \\
\text { responsive }\end{array}$ & 10 & Responsive & 10 & 3738 \\
\hline
\end{tabular}

nodes for all the datasets are given in Appendix B and the significance of these nodes obtained from the values are discussed further in the results.

\subsection{Algorithmic Complexity}

The algorithmic complexity of the given dynamics is $O\left(M^{2}\right)$ as each gene is compared with every other gene to produce a similarity matrix of size $m \times m$. Using a non-linear differential equation can only process datasets with limited number of genes. But, our model is capable of processing few thousand genes and hence this complexity is computationally less when compared to other methods that use differential equations. If, when combined with a higher processor speed, this method can be applied to even larger datasets to find a broader classification of target genes for experimental analysis.

\section{Results and Discussion}

The method has been applied to three different datasets: experimental rhinovirus infections in humans, atopic CD4 $\mathrm{T}$ cell responses to allergens, and responses to cancer immunotherapy in mice. The algorithm is robust in identifying the high betweenness nodes for different organisms studied under different experimental conditions. The provided datasets have a set of genes that show significant change with respect to the controls for the respective sub-ject of study; the rest have been deemed non-informative and discarded.

The distribution of genes in a network is based on correlation properties like structural, functional and metabolism dictated by the dynamics applied to the expression data. In our case, we obtain a functional network where, the nodes correspond to the genes that are highly influential. With a complexity of $O\left(M^{2}\right)$, even though the size of dataset is limited to less than 10000 genes or nodes, it is still sufficient to identify target nodes that can be further evaluated experimentally. We apply a novel method, which, identifies gene nodes dynamically in different datasets.

Betweenness centrality of the nodes indicate that the deletion of the highly connected nodes will bring maximum change in the expression of other genes or nodes. By building an adjacency matrix using a threshold from the similarity matrix $\mathbf{P}$ and then applying betweenness centrality to the graph of the adjacency matrix, we obtain the high betweenness centrality nodes. Some of these nodes have known biological significance with respect to their function and some are novel nodes which can be potentially explored in follow-up mechanistic studies [Bosco et al., 2016]. We computationally validate the significance of these nodes and their corresponding edges using URA. For each of the top 10 high betweenness centrality node, we take all the nodes that are connected to that node and apply IPA-URA. We analyze if the high betweenness centrality node as determined by our algorithm is indeed an upstream regulator for the given set of edges connected to the node using IPA-URA, as URA contains a database of all the experimentally determined relationships between upstream regulators and downstream targets [Kaneko et al., 2013]. We also look at the top upstream regulator for the given set of edges as deemed by the IPA-URA; the top regulator is also provided in Appendix B. 
Table 3: The list of top 10 high betweenness centrality nodes for allergy, rhinovirus infection and cancer immunotherapy.

\begin{tabular}{|l|l|l|l|l|l|l|}
\hline No. & Allergy & $\begin{array}{l}\text { BC } \\
\text { Value }\end{array}$ & $\begin{array}{l}\text { Rhinovirus } \\
\text { Infection }\end{array}$ & $\begin{array}{l}\text { BC } \\
\text { Value }\end{array}$ & $\begin{array}{l}\text { Cancer } \\
\text { Immuno- } \\
\text { therapy }\end{array}$ & $\begin{array}{l}\text { BC } \\
\text { Value }\end{array}$ \\
\hline 1 & PLBD1 & 39666.45 & LINC01279 & 109490.86 & Amigo2 & 37509.63 \\
2 & SERPINB2 & 38788.83 & KRT6A & 43819.47 & Adcy8 & 11476.49 \\
3 & IL1 $\beta$ & 13728.00 & GXYLT2 & 27578.13 & Gm773 & 10804.81 \\
4 & GPNMB & 11190.40 & KRT6C & 8369.60 & Flnb & 3777.63 \\
5 & TGFBI & 9097.35 & SERPINB11 & 5246.88 & Mcpt2 & 3309.41 \\
6 & CXCL10 & 6465.83 & DLEC1 & 4182.80 & Akr1b8 & 2403.83 \\
7 & ME1 & 4918.81 & COX7A1 & 2884.95 & Slc25a24 & 2163.56 \\
8 & LYZ & 1702.94 & ARMC2 & 1954.09 & $2700094-$ & 2073.34 \\
9 & & & & & K13Rik & \\
10 & ALDH1A1 & 1662.49 & SEC14L3 & 1880.52 & Cdh2 & 557.85 \\
RNASE6 & 1218.49 & CCDC81 & 1507.40 & Glud1 & 230.26 \\
\hline
\end{tabular}

Some of the target nodes that have higher betweenness centrality values are discussed in this section to elucidate the importance of these nodes and how they can be used as potential bio-markers for the subject of study. The size of the network is dictated by the threshold used to build the adjacency matrix; the threshold is chosen by applying node entropy to the adjacency matrix. For a range of different thresholds, we find the optimum point at which the change in entropy is non-trivial and use value as threshold to build the network. The higher the threshold, the lesser the number of target nodes. If the threshold is lowered, there are more target nodes which gives wider range of targets rather than selected few. The top 10 high betweenness centrality nodes are given in Table 2 .

Gene information, associated pathways and the potential upstream regulators are given in section Appendix $\mathrm{B}$ for the top high betweenness centrality nodes. Among the list of nodes that are given in Table 2, for allergy, IL1 $\beta$, GPNMB, CXCL10, LYZ and for cancer immunotherapy, 2700094K13Rik (SelenoH) have been identified as upstream regulators to the given set of nodes that are connected to them by the algorithm. There are no nodes that have been identified as upstream regulators for Rhinovirus infection. There exists proven connection between some of the connected nodes and the high betweenness centrality nodes according to IPA-URA ranking, even though they are not the top ranking upstream regulators. The rest of the high betweenness centrality nodes could be potential novel targets that can be further analyzed in mechanistic studies [Bosco et al., 2016]. For the given set of nodes connected to each of the high betweenness centrality node, the genes or pathways mentioned in the tables of section Appendix $\mathrm{B}$ are determined to be the upstream regulators by the IPA-URA. Majority of the high betweenness centrality nodes of allergy dataset has IFNG as upstream regulator and for Rhinovirus infection, it is either IFNG or $I F N-\alpha$; cancer immunotherapy had TP53 as upstream regulator, for most of the genes. The list of genes that are connected to the high betweenness centrality nodes are enriched with antiviral or immune signaling molecules for rhinovirus and allergy datasets and in case of cancer immunotherapy, it is the tumor suppressors.

The high betweenness centrality nodes with upstream regulators identified by IPA have known experiemntal evidences that link them to their study of interest. Some of the validated relations are explained as follows. CXCL10 is up-regulated in pulmonary infections and is known to have defensin-like anti-microbial activity [Porter et al., 2008]. IL1 $\beta$ is a known pro-inflammatory gene that is expressed in the airway smooth muscles and can increase inflammatory response during altered airway response [Whelan et al., 2004]. GPNMB is also a pro-inflammatory signaling molecule [Ripoll et al., 2007] and gets down-regulated upon onset of asthma [Bergauer et al., 2017]. LYZ is one of the genes that get down-regulated in eosinophil-associated disorders, including asthma [Shen et al., 2015]. Increased expression of SelenoH gene has been demonstrated to accelerate the supression of P53 pathway leading to apoptosis [Mendelev et al., 2009, Mendelev et al., 2011]. These nodes have been shown to have links with their corresponding studies of interest while the rest of the nodes are novel; they can be potential bio-markers to identify the onset of disease or can be used as targets for experimental validation. 
Figure 2: The plot of top 10 high betweenness centrality nodes and their corresponding values for allergy, rhinovirus infection and cancer immunotherapy from the table 4 . The plot shows the steep decrease in betweenness centrality, when they are ordered from the highest to the lowest values as ranked by the algorithm.

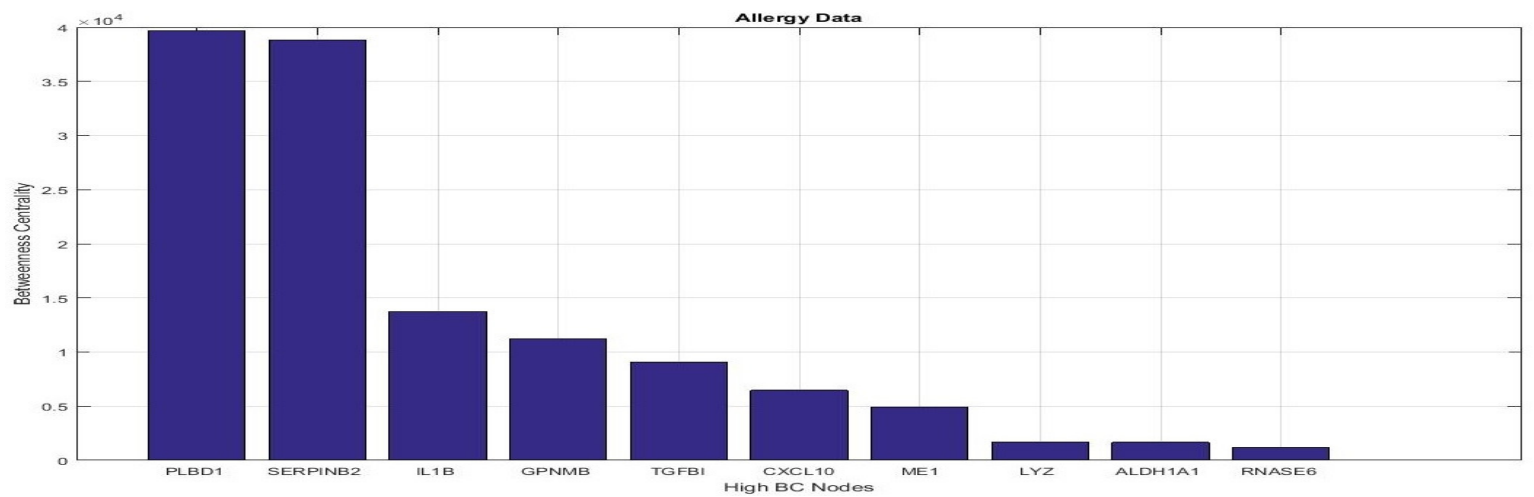

2a. BC plot for Allergy Data



2b. BC plot for Rhinovirus Infection Data

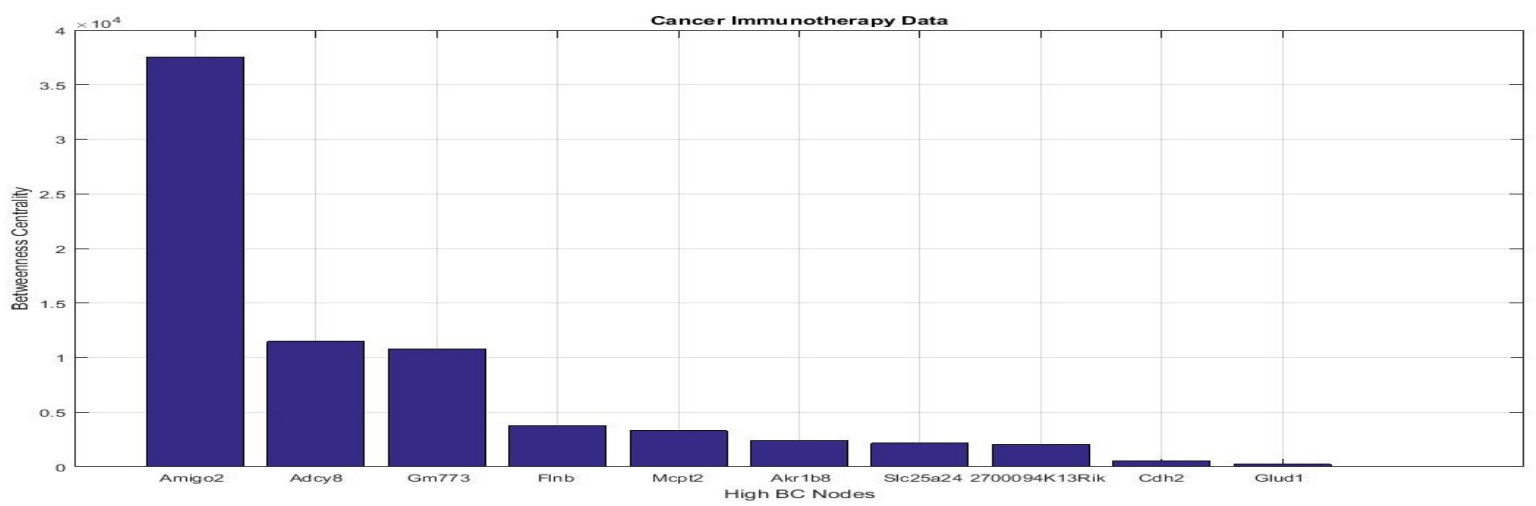

2c. BC plot for Cancer Immunotherapy data

The datasets that have been processed through the algorithm contains most of the differentially expressed genes for their respective subject of study. As the betweenness centrality values denote (Table 2 ), the values are descending. We analyze the top 10 high betweenness centrality nodes in assumption that they have more significance in regulating the system. It is surprising to note that the edges connected to these nodes are enriched with genes associated with antiviral properties for allergy dataset and the top upstream regulator for most of the nodes being IFN $\gamma$, IL4, CTSG and CREB- $\kappa$ F. Asthma, especially when triggered by allergen exposure tends to activate the Th1 and Th2 pathways which show considerable change with airway inflammation [Shum et al., 2006], cytokines and several chemokines [Holgate et al., 2009] and our analysis is consistent with the prior findings. The rhinovirus infection has 
wide range of upstream regulators: IFN $\gamma$, IFN $\alpha$, IFNA10, STAT1 and TLR3 and these are all components of the interferon-mediated innate antiviral response that is essential for preventing the spread of viral infections [Bosco et al., 2012]. Similarly, the cancer immunotherapy nodes are enriched with genes that are regulated by TP53, IL4 and IL15. The number of high betweenness centrality nodes that have known prior association with the study of interest is not consistent across datasets, while we do not observe any potential upstream regulators for Rhinovirus infection dataset. One possible explanation for could be that the high betweenness centrality nodes in the rhinovirus dataset are integrating signals downstream of the upstream regulators and this would require further analyses to explore. The list of all top upstream regulators and the actual function of the genes according to GO annotation and GeneCards are given in Appendix B. The aim of this manuscript is to focus on applying a novel method to find the high betweenness centrality nodes, that could be used as potential targets for experimental analysis. Even though, the betweenness centrality is used to build a network of genes, we are not analysing the structure and properties of the network. It is beyond the scope of this manuscript and is a path for further studies.

The algorithm does indeed identify nodes that have known links for the other datasets. The verification of the results was done using IPA-URA, a bioinformatics program that infers upstream regulators that can either activate or inhibit a set of genes by searching for links between genes and their functions in the experimental biomedical literature[Kaneko et al., 2013]. The use of this algorithm in identifying nodes that regulating the rest of the system is a potential future exploration pathway for mechanistic studies. If the nodes can be utilized as bio-markers to identify the onset of disease has to be further explored. The method is computational; even though some of the nodes have been proven to have significant impact on the study of interest, the validation of this model is performed using IPA-URA, the literature repository for all experimental studies. Given that, the model can identify both node genes that have known associations and novel genes, and therefore, can identify candidate for mechanistic or drug development programs.

\section{Conclusion}

Biological systems are complex and the genomic data obtained typically contain a large number of variables and a small number of samples. The data is largely influenced by the experimental context and conditions, and the tissue type. The aim of using mathematical models is to identify biologically relevant targets and thus, prioritize candidates for further experimentation. We have used an algorithm based on a differential equation using three terms a basal level expression, degradation and change in expression governed by Michaelis-Menten kinetics. This method has helped us identify some useful targets for all datasets, some of which have already been under study and additionally some novel gene targets. The upstream regulatory analysis performed using IPA also demonstrates that the potential targets are associated to the pathways that are relevant to the study of interest. We emphasize that the top high betweenness centrality nodes have been validated using a computational methods, IPAURA and not been specifically validated in the same experimental system, as IPA-URA is a repository of experimentally determined molecular relationships from diverse experimental conditions. We propose the rest of the high betweenness centrality nodes as potential targets or bio-markers that can be explored in the future.The algorithm has only been applied to three datasets from diverse experimental settings, and on a large scale, the accuracy of the results are yet to be proven. Also, if chosen as bio-markers, the efficiency of the nodes as target and therapeutic role have to be assessed using further studies. The distance measured by the algorithm is based on the expression values for the subject of study. None of the values are assumed or referred from the literature, hence, the resulting network is driven completely by the actual expression values. Therefore, environmental conditions under which the experiments are performed will have a greater impact on the resulting nodes. Even though the method can process few thousand genes, it is still lesser compared to other methods like Boolean or Bayesian networking and hence some pre-filtering of the variables is required to use the algorithm. Also, there needs to be at least one differentially expressed state of the genes be present to find the change in expression. Despite these limitations, we have been able to find important nodes without prior knowledge of all the genes involved using the algorithm. 


\section{Further Studies}

The method could be further developed such that it can be applied to larger datasets and not limited to just few thousands of genes. Also we have only considered the effect of one gene on another. We have not taken into account the effect of more than one gene on an expression. We also forgo the differentiation between transcription factors and non-transcription factors, which can further change the results. We are also using only a single time point from the dataset; the application to a time series analysis with samples collected over a period of time can also change the outcome of the algorithm. These aspects are potential future scopes for this algorithm.

\section{Funding}

This work has been supported by Australian Research Council Linkage Grant LP130101055. Anthony Bosco is supported by a Fellowship from Simon Lee Foundation.

\section{References}

[Abdi et al., 2010] Hervé Abdi and Lynne J Williams. Principal component analysis. Wiley Interdisciplinary Reviews: Computational Statistics, 2(4):433-459, 2010.

[Alvarez et al., 2016] Mariano J Alvarez, Yao Shen, Federico M Giorgi, Alexander Lachmann, B Belinda Ding, B Hilda Ye, and Andrea Califano. Functional characterization of somatic mutations in cancer using network-based inference of protein activity. Nature Genetics, 2016.

[Barabási et al., 2011] Albert-László Barabási, Natali Gulbahce, and Joseph Loscalzo. Network medicine: a network-based approach to human disease. Nature Reviews Genetics, 12(1):56-68, 2011.

[Berg et al., 2002] Jeremy M Berg, John L Tymoczko, and Lubert Stryer. Enzymes: Basic concepts and kinetics. 2002.

[Bergauer et al., 2017] Annika Bergauer, Nina Sopel, Bettina Kroß, Tytti Vuorinen, Paraskevi Xepapadaki, Scott T Weiss, Ashley Blau, Himanshu Sharma, Cornelia Kraus, Rebekka Springel, Manfred Rauh, Susanne Mittler, Anna Graser, Theodor Zimmermann, Volker O Melichar, Alexander Kiefer, Marek L Kowalski, Anna Sobanska, Tuomas Jartti, Heikki Lukkarinen,10, Nikolaos G Papadopoulos and Susetta Finotto. IFN- $\alpha / \mathrm{IFN}-\lambda$ responses to respiratory viruses in paediatric asthma. European Respiratory Journal, 49(2):1600969, 2017.

[Bhakta et al., 2018] Sunil Bhakta, Lisa M. Crocker, Yvonne Chen, Meredith Hazen, Melissa M. Schutten, Dongwei Li, Coenraad Kuijl, Rachana Ohri, Fiona Zhong, Kirsten A. Poon, Mary Ann T. Go, Eric Cheng, Robert Piskol, Ron Firestein, Aimee Fourie-O'Donohue, Katherine R. Kozak, Helga Raab, Jo-Anne Hongo, Deepak Sampath, Mark S. Dennis, Richard H. Scheller, Paul Polakis and Jagath R. Junutula An Anti-GDNF Family Receptor Alpha 1 (GFRA1) Antibodyâ"Drug Conjugate for the Treatment of Hormone Receptorâ"Positive Breast Cancer Molecular Cancer Therapeutics, 17(3):638$649,2018$.

[Bosco et al., 2012] Anthony Bosco, Samira Ehteshami, Sujatha Panyala, Fernando D Martinez. Interferon regulatory factor 7 is a major hub connecting interferon-mediated responses in virus-induced asthma exacerbations in vivo. Journal of Allergy and Clinical Immunology, 129(1):88-94, 2012.

[Bosco et al., 2016] Anthony Bosco, Shahina Wiehler and David Proud. Interferon regulatory factor 7 regulates airway epithelial cell responses to human rhinovirus infection. BMC Genomics, 17(1):76, 2016.

[Chan et al., 2008] Zeke SH Chan, Ilkka Havukkala, Vishal Jain, Yingjie Hu, and Nikola Kasabov. Soft computing methods to predict gene regulatory networks: An integrative approach on time-series gene expression data. Applied Soft Computing, 8(3):1189-1199, 2008.

[de Silva et al., 2005] Eric de Silva and Michael PH Stumpf. Complex networks and simple models in biology. Journal of the Royal Society Interface, 2(5):419-430, 2005. 
[Dittmar et al., 2002] Gunnar A. G. Dittmar, Caroline R. M. Wilkinson, Paul T. Jedrzejewski and Daniel Finley. Role of a ubiquitin-like modification in polarized morphogenesis. AAAS Science, 295(5564):2442-2446, 2006.

[Feizi et al., 2013] Soheil Feizi, Daniel Marbach, Muriel Médard, and Manolis Kellis. Network deconvolution as a general method to distinguish direct dependencies in networks. Nature Biotechnology, 31(8):726-733, 2013.

[Friedman et al., 2000] Nir Friedman, Michal Linial, Iftach Nachman, and Dana Pe'er. Using Bayesian networks to analyze expression data. Journal of Computational Biology, 7(3-4):601-620, 2000.

[Hecker et al., 2009] Michael Hecker, Sandro Lambeck, Susanne Toepfer, Eugene Van Someren, and Reinhard Guthke. Gene regulatory network inference: data integration in dynamic models-a review. Biosystems, 96(1):86-103, 2009.

[Holgate et al., 2009] Stephen T Holgate and Donna E Davies. Rethinking the pathogenesis of asthma. Immunity, 31(3):362-367, 2009.

[Ingalls et al., 2013] Brian Ingalls. Mathematical modelling in systems biology: An introduction. Internet.[cited at p. 117], 2013.

[Janes et al., 2006] Kevin A Janes and Michael B Yaffe. Data-driven modelling of signal-transduction networks. Nature Reviews Molecular Cell Biology, 7(11):820-828, 2006.

[Junttila et al., 2005] Teemu T Junttila, Maria Sundvall, Mikael Lundin, Johan Lundin, Minna Tanner, Pirkko Härkönen, Heikki Joensuu, Jorma Isola and Klaus Elenius. Cleavable ErbB4 isoform in estrogen receptor-regulated growth of breast cancer cells. Cancer Research, 65(4):1384-1393, 2005.

[Kaneko et al., 2013] Yoskiko Kaneko, Yohei Yatagai, Hideyasu Yamada, Hiroki Iijima, Hironori Masuko, Tohru Sakamoto and Noduyuki Hizawa. The search for common pathways underlying asthma and COPD. International Journal of chronic obstructive pulmonary disease, 8:65, 2013.

[Képés et al., 2007] François Képès. Biological networks. World Scientific, 2007.

[Kim et al., 2011] Mi Hyeon Kim, Hwa Jeong Seo, Je-Gun Joung, and Ju Han Kim. Comprehensive evaluation of matrix factorization methods for the analysis of dna microarray gene expression data. BMC Bioinformatics, 12(13):1, 2011.

[Kong et al., 2011] Wei Kong, Xiaoyang Mou, and Xiaohua Hu. Exploring matrix factorization techniques for significant genes identification of Alzheimerât ${ }^{\mathrm{TM}} \mathrm{S}$ disease microarray gene expression data. BMC Bioinformatics, 12(5):1, 2011.

[Kossenkov et al., 2010] Andrew V Kossenkov and Michael F Ochs. Matrix factorisation methods applied in microarray data analysis. International journal of Data Mining and Bioinformatics, 4(1):72-90, 2010 .

[Kulkarni et al., 2016] Vidyadhar G Kulkarni. Modeling and analysis of stochastic systems. CRC Press, 2016.

[Lee et al., 2012] Chien-Pang Lee, Yungho Leu, and Wei-Ning Yang. Constructing gene regulatory networks from microarray data using ga/pso with dtw. Applied Soft Computing, 12(3):1115-1124, 2012.

[Lee et al., 2009] Wei-Po Lee and Wen-Shyong Tzou. Computational methods for discovering gene networks from expression data. Briefings in Bioinformatics, 10(4):408-423, 2009.

[Lesterhuis et al., 2015] W Joost Lesterhuis, Catherine Rinaldi, Anya Jones, Esdy N Rozali, Ian M Dick, Andrea Khong, Louis Boon, Bruce W Robinson, Anna K Nowak, Anthony Bosco and Richard A. Lake. Network analysis of immunotherapy-induced regressing tumours identifies novel synergistic drug combinations, Scientific Reports, Nature Publishing Group, 5:12298, 2015.

[Mendelev et al., 2009] Natalia Mendelev, Sam Witherspoon and P Andy Li. Overexpression of human selenoprotein $\mathrm{H}$ in neuronal cells ameliorates ultraviolet irradiation-induced damage by modulating cell signaling pathways. Experimental neurology, 220(2):328-334, 2009. 
[Mendelev et al., 2011] Natalia Mendelev, Suresh L Mehta, Sam Witherspoon, Qingping He, Jonathan Z Sexton and P Andy Li. Upregulation of human selenoprotein $\mathrm{H}$ in murine hippocampal neuronal cells promotes mitochondrial biogenesis and functional performance. Mitochondrion, 11(1):76-82, 2011.

[Moloshok et al., 2002] Thomas D Moloshok, RR Klevecz, Jeffrey D Grant, Frank J. Manion, WFT Speier, and Michael F. Ochs. Application of Bayesian decomposition for analysing microarray data. Bioinformatics, 18(4):566-575, 2002.

[Montllor et al., 2019] Claudia Montllor-Albalate, Alyson E Colin, Bindu Chandrasekharan, Naimah Bolaji, Joshua L Anderson, Wayne F Outten and Amit R Reddi. Extra-mitochondrial $\mathrm{Cu} / \mathrm{Zn}$ superoxide dismutase (Sod1) is dispensable for protection against oxidative stress but mediates peroxide signaling in Saccharomyces cerevisiae. Radox Biology, 21, 2019.

[Palacios-Arreola et al., 2014] Isabel M Palacios-Arreola, Karen E Nava-Castro, Julieta I Castro, Eduardo García-Zepeda, Julio C Carrero and Jorge Morales-Montor. The role of chemokines in breast cancer pathology and its possible use as therapeutic targets. Journal of Immunology Research, 2014.

[Panse et!al., 2008] Panse, J Panse and Friedrichs, K Friedrichs, A Marx, Y Hildebrandt, T Luetkens, K Bartels, C Horn, T Stahl, Y Cao, K Milde-Langosch and A Niendorf. Chemokine CXCL13 is overexpressed in the tumour tissue and in the peripheral blood of breast cancer patients. British Journal of Cancer, 99(6):930, 2008.

[Porter et al., 2008] Joanna C Porter, Mary Falzon, and Alan Hall. Polarized localization of epithelial CXCL11 in chronic obstructive pulmonary disease and mechanisms of $\mathrm{T}$ cell regression. The Journal of Immunology, 180(3):1866-1877, 2008.

[Proud et al., 2008] David Proud, Ronald B Turner, Birgit Winther, Shahina Wiehler, Jay P Tiesman, Tim D Reichling, Kenton D Juhlin, Andy W Fulmer, Begonia Y Ho, Amy A Walanski, Cathy L Poore, Haruko Mizoguchi, Lynn Jump, Marsha L Moore, Claudine K Zukowski and Jeffrey W Clymer. Gene expression profiles during in vivo human rhinovirus infection Am J Respir Crit Care Med, 178:962-968, 2008.

[Ripoll et al., 2007] Vera M Ripoll, Katharine M Irvine, Timothy Ravasi, Matthew J Sweet and David A. Hume. Gpnmb is induced in macrophages by IFN- $\gamma$ and lipopolysaccharide and acts as a feedback regulator of proinflammatory responses Am Assoc Immnol, 178(10):6557-6566, 2007.

[Ronen et al., 2002] Micha Ronen, Revital Rosenberg, Boris I Shraiman, and Uri Alon. Assigning numbers to the arrows: Parameterizing a gene regulation network by using accurate expression kinetics. Proceedings of the National Academy of Sciences, 99(16):10555-10560, 2002.

[Sacchi et al., 2007] Lucia Sacchi, Cristiana Larizza, Paolo Magni, and Riccardo Bellazzi. Precedence temporal networks to represent temporal relationships in gene expression data. Journal of Biomedical Informatics, 40(6):761-774, 2007.

[Shen et al., 2015] Zhong-Jian Shen, Jie Hu, Stephane Esnault, Igor Dozmorov and James S Malter. RNA Seq profiling reveals a novel expression pattern of TGF- $\beta$ target genes in human blood eosinophils. Immunology letters, 167(1):1-10, 2015.

[Shum et al., 2006] Bennett OV Shum, Charles R Mackay, Cem Z Gorgun, Melinda J Frost, Rakesh K Kumar, Gökhan S Hotamisligil, and Michael S Rolph. The adipocyte fatty acid-binding protein AP2 is required in allergic airway inflammation. The Journal of Clinical Investigation, 116(8):2183-2192, 2006.

[Troy et al., 2016] Niamh M Troy, Elysia M Hollams, Patrick G Holt and Anthony Bosco. Differential gene network analysis for the identification of asthma-associated therapeutic targets in allergen-specific T-helper memory responses. BMC Medical Genomics, 9(1), 2016.

[Wang et al., 2014] YX Rachel Wang and Haiyan Huang. Review on statistical methods for gene network reconstruction using expression data. Journal of Theoretical Biology, 362:53-61, 2014.

[Wang et al., 2013] Jianxin Wang, Xiaoqing Peng, Min Li, and Yi Pan. Construction and application of dynamic protein interaction network based on time course gene expression data. Proteomics, 13(2):301$312,2013$. 
[Whelan et al., 2004] R Whelan, C Kim, M Chen, J Leiter, MM Grunstein, and H Hakonarson. Role and regulation of Interleukin-1 molecules in pro-asthmatic sensitised airway smooth muscle. European Respiratory Journal, 24(4):559-567, 2004.

[White et al., 1994] Douglas R White and Stephen P Borgatti. Betweenness centrality measures for directed graphs. Social Networks, 16(4):335-346, 1994.

[Yang et al., 2012] Ivana V Yang, John Tomfohr, Jaspal Singh, Catherine M Foss, Harvey E Marshall, Loretta G Que, Erin McElvania-Tekippe, Sarita Florence, John S Sundy, and David A Schwartz. The clinical and environmental determinants of airway transcriptional profiles in allergic asthma. American Journal of Respiratory and Critical Care Medicine, 185(6):620-627, 2012.

[Zou et al., 2004] Min Zou and Suzanne D Conzen. A new dynamic Bayesian network (dbn) approach for identifying gene regulatory networks from time course microarray data. Bioinformatics, 21(1):71-79, 2004.

[1] http://www.sci.unich.it/ francesc/teaching/network/betweeness.html.

[2] http://med.bioinf.mpi-inf.mpg.de/netanalyzer/help/2.7/.

[3] http://pages.ingenuity.com/rs/ingenuity/images/0812\\%20upstream_regulator_analysis_ whitepaper.pdf. 


\section{Appendix A}

\section{Michaelis-Menten Kinetics}

In general, Michaelis-Menten Kinetics (MMK) deal with the irreversible binding of an enzyme to a substrate to form products. The enzyme reversibly binds to the substrate to form product [Berg et al., 2002].

$$
E+S \underset{k_{-1}}{\stackrel{k_{1}}{\rightleftharpoons}} E S \stackrel{k_{2}}{\rightarrow} E+P
$$

Where $\mathrm{E}$ is the enzyme, $\mathrm{S}$ is the substrate, $\mathrm{ES}$ is the enzyme-substrate complex, $k_{1}$ is the rate of formation of ES complex, $k_{-1}$ is the rate of dissociation of ES complex and $k_{2}$ is the rate of product formation from ES complex.

The total rate of forward reaction is equal to the rate of reverse reaction and the concentration of the enzyme remains constant throughout the reaction. The amount of free enzyme is equal to the difference between the total enzyme and the enzyme bound to the substrate [Berg et al., 2002].



Figure 3: Michaelis-Menten Kinetics portraying the rate of product formation.

$$
\frac{d[E S]}{d t}=k_{1}[E] \times[S]-k_{-1} \times[E S]-k_{2} \times[E S]
$$

The rate of formation of the product (velocity of product formation) is given by,

$$
v=\frac{d[P]}{d t}=k_{2} \times[E S]=\frac{k_{2} \times E_{0} \times[S]}{K_{m}+[S]}=\frac{V_{m} \times[S]}{K_{m}+[S]}
$$

where,

$$
K_{m}=\frac{k_{-1}}{k_{1}}=\frac{[E][S]}{[E S]} \text { and }
$$

$K_{m}$ is the Michaelis-Menton constant at which the substrate concentration at half of the maximum velocity.

$$
V_{m}=k_{2} \times E_{0}
$$

The enzyme level remains constant and $E_{0}$ is the initial enzyme concentration.

$$
E=E_{0}-[E S]
$$

$V_{m}$ is the maximum production rate where the enzyme is completely saturated with the substrates to convert it into product. 


\section{Appendix B}

The 10 high betweenness centrality nodes for each of the datasets and their gene information and associated pathways as per GO annotations and Gene Card dataset are mentioned below. Also mentioned are their corresponding top upstream regulators as obtained from IPA-URA for each of the nodes.

Table 4: Betweenness centrality for Allergy Dataset

\begin{tabular}{|c|c|c|c|c|}
\hline No. & Gene ID & Gene Information & Pathways (GO/GeneCards) & $\begin{array}{l}\text { IPA-URA } \\
\text { Upstream } \\
\text { Regulator }\end{array}$ \\
\hline 1 & PLBD1 & $\begin{array}{l}\text { Phospholipase B } \\
\text { Domain Containing } 1\end{array}$ & $\begin{array}{l}\text { Glycerophospholipid } \\
\text { biosynthesis and Metabolism. } \\
\text { Hydrolase activity }\end{array}$ & IFNG \\
\hline 2 & SERPINB2 & $\begin{array}{l}\text { Serpin Family } \\
\text { B Member } 2\end{array}$ & $\begin{array}{l}\text { Complement and coagulation } \\
\text { cascades and Response to } \\
\text { elevated platelet cytosolic } \\
\text { Ca2+. Serine-type endo- } \\
\text { peptidase inhibitor activity. }\end{array}$ & CTSG \\
\hline 3 & IL1B & Interleukin 1 Beta & $\begin{array}{l}\text { PEDF Induced Signaling } \\
\text { and Bacterial infections in } \\
\text { CF airways. Protein domain } \\
\text { specific binding } \\
\text { and interleukin-1 } \\
\text { receptor binding }\end{array}$ & CREB-KF \\
\hline 4 & GPNMB & Glycoprotein Nmb & $\begin{array}{l}\text { Signaling by GPCR and } \\
\text { Signaling by PTK } 6 . \\
\text { Heparin binding and } \\
\text { integrin binding. }\end{array}$ & IFNG \\
\hline 5 & TGFBI & $\begin{array}{l}\text { Transforming Growth } \\
\text { Factor Beta Induced }\end{array}$ & $\begin{array}{l}\text { Activated PKN1 stimulates } \\
\text { transcription of AR (androgen } \\
\text { receptor) regulated genes } \\
\text { KLK2 and KLK3 and Metabolism } \\
\text { of proteins. Integrin binding } \\
\text { and extracellular matrix binding. }\end{array}$ & IFNG \\
\hline 6 & CXCL10 & $\begin{array}{l}\text { C-X-C Motif } \\
\text { Chemokine Ligand } 10\end{array}$ & $\begin{array}{l}\text { PEDF Induced Signaling } \\
\text { and Cytokine Signaling in } \\
\text { Immune system. Receptor } \\
\text { binding and chemokine activity. }\end{array}$ & IFNG \\
\hline 7 & ME1 & Malic Enzyme 1 & $\begin{array}{l}\text { Metabolism and Regulation } \\
\text { of lipid metabolism by } \\
\text { Peroxisome proliferator- } \\
\text { activated receptor alpha } \\
\text { (PPARalpha). Electron transfer } \\
\text { activity and NAD binding. }\end{array}$ & IFNG \\
\hline 8 & LYZ & Lysozyme & $\begin{array}{l}\text { Innate Immune System } \\
\text { and Activated PKN1 stimulates } \\
\text { transcription of AR (androgen } \\
\text { receptor) regulated genes KLK2 } \\
\text { and KLK3. Identical protein } \\
\text { binding and lysozyme activity. }\end{array}$ & IL4 \\
\hline 9 & ALDH1A1 & $\begin{array}{l}\text { Aldehyde } \\
\text { Dehydrogenase } 1 \\
\text { Family Member A1 }\end{array}$ & $\begin{array}{l}\text { Glycosaminoglycan metabolism } \\
\text { and Drug metabolism - } \\
\text { cytochrome P } 450 \text {. } \\
\text { Oxidoreductase activity and } \\
\text { acyl-CoA dehydrogenase activity. }\end{array}$ & IFNG \\
\hline 10 & RNASE6 & $\begin{array}{l}\text { Ribonuclease A } \\
\text { Family Member K6 }\end{array}$ & $\begin{array}{l}\text { Innate Immune System } \\
\text { and Defensins. Nucleic acid }\end{array}$ & IFNG \\
\hline
\end{tabular}


Table 5: Betweenness centrality for Rhinovirus Infection Dataset

\begin{tabular}{|c|c|c|c|c|}
\hline No. & Gene ID & Gene Information & Pathways (GO/GeneCards) & $\begin{array}{l}\text { IPA-URA } \\
\text { Upstream } \\
\text { Regulator }\end{array}$ \\
\hline 1 & LINC01279 & $\begin{array}{l}\text { Long Intergenic } \\
\text { Non-Protein } \\
\text { Coding RNA } 1279\end{array}$ & Non-coding RNA & IFNG \\
\hline 2 & KRT6A & Keratin $6 \mathrm{~A}$ & $\begin{array}{l}\text { Ectoderm Differentiation } \\
\text { and Keratinization }\end{array}$ & IFNA10 \\
\hline 3 & GXYLT2 & $\begin{array}{l}\text { Glucoside } \\
\text { Xylosyl- } \\
\text { transferase } 2\end{array}$ & $\begin{array}{l}\text { O-glycan biosynthesis } \\
\text { Transferase activity, transferring } \\
\text { glycosyl groups and } \\
\text { UDP-xylosyltransferase } \\
\text { activity }\end{array}$ & $\begin{array}{l}\text { Interferon } \\
\text { alpha }\end{array}$ \\
\hline 4 & KRT6C & Keratin $6 \mathrm{C}$ & $\begin{array}{l}\text { Keratinization and } \\
\text { Developmental } \\
\text { activity. Structural molecule } \\
\text { Activity }\end{array}$ & IFNA10 \\
\hline 5 & SERPINB11 & $\begin{array}{l}\text { Serpin Family } \\
\text { B Member } 11 \\
\text { (Gene/Pseudogene) }\end{array}$ & $\begin{array}{l}\text { Serine-type endo- } \\
\text { peptidase inhibitor } \\
\text { activity }\end{array}$ & STAT1 \\
\hline 6 & DLEC1 & $\begin{array}{l}\text { Deleted In Lung } \\
\text { And Esophageal } \\
\text { Cancer } 1\end{array}$ & $\begin{array}{l}\text { Eosophageal cancer and } \\
\text { Lung cancer }\end{array}$ & IFNG \\
\hline 7 & COX7A1 & $\begin{array}{l}\text { Cytochrome C } \\
\text { Oxidase Subunit } \\
7 \mathrm{~A} 1\end{array}$ & $\begin{array}{l}\text { Respiratory electron transport, } \\
\text { ATP synthesis by } \\
\text { chemisosmotic coupling, } \\
\text { heat production by } \\
\text { uncoupling proteins, AMPK } \\
\text { enzyme coupling activity. } \\
\text { Electron transfer and } \\
\text { cytochrome C-oxidase activity. }\end{array}$ & $\begin{array}{l}\text { Interferon } \\
\text { alpha }\end{array}$ \\
\hline 8 & ARMC2 & $\begin{array}{l}\text { Armadillo Repeat } \\
\text { Containing } 2\end{array}$ & Binding & STAT1 \\
\hline 9 & SEC14L3 & $\begin{array}{l}\text { SEC14 Like } \\
\text { Lipid Binding } 3\end{array}$ & $\begin{array}{l}\text { Transporter activity and } \\
\text { Lipid binding }\end{array}$ & IFNG \\
\hline 10 & CCDC81 & $\begin{array}{l}\text { Coiled-Coil } \\
\text { Domain }\end{array}$ & Protein-coding gene & TLR3 \\
\hline
\end{tabular}


Table 6: Betweenness centrality for Cancer Immunotherapy Dataset

\begin{tabular}{|c|c|c|c|c|}
\hline No. & Gene ID & Gene Information & Pathways (GO/GeneCards) & $\begin{array}{l}\text { IPA-URA } \\
\text { Upstream } \\
\text { Regulator }\end{array}$ \\
\hline 1 & Amigo2 & $\begin{array}{l}\text { Adhesion molecule } \\
\text { with Ig like } \\
\text { domain } 2\end{array}$ & $\begin{array}{l}\text { Cell death, } \\
\text { cellular component } \\
\text { orientation }\end{array}$ & IL15 \\
\hline 2 & Adcy8 & $\begin{array}{l}\text { Adenylate } \\
\text { cyclase } 8\end{array}$ & $\begin{array}{l}\text { Carbohydrate derivative } \\
\text { metabolism, response to } \\
\text { stimulus signalling }\end{array}$ & TP53 \\
\hline 3 & $\mathrm{Gm} 773$ & Predicted gene 773 & & TP53 \\
\hline 4 & Flnb & Filamin, beta & $\begin{array}{l}\text { Cellular differentiation, } \\
\text { cellular component } \\
\text { organization, immune system } \\
\text { process, response to } \\
\text { stimulus, system development }\end{array}$ & TP53 \\
\hline 5 & Mcpt2 & Cell protease 2 & Protein metabolic process & TP53 \\
\hline 6 & Akr1b8 & $\begin{array}{l}\text { Aldo-keto } \\
\text { reductase family } \\
1, \text { member B } 8\end{array}$ & $\begin{array}{l}\text { Carbohydrate derivative } \\
\text { metabolism, lipid } \\
\text { metabolic process }\end{array}$ & TP53 \\
\hline 7 & Slc25a24 & $\begin{array}{l}\text { Solute carrier } \\
\text { family } 25 \\
\text { (mitochondrial } \\
\text { carrier, phospate } \\
\text { carrier), member } 24\end{array}$ & $\begin{array}{l}\text { Cell death, } \\
\text { establishment localization, } \\
\text { response to stimulus }\end{array}$ & TP53 \\
\hline 8 & 2700094K13Rik & Selenoprotein H & & TP53 \\
\hline 9 & Cdh2 & Cadherin 2 & $\begin{array}{l}\text { Differentiation, proliferation, } \\
\text { cellular component organization, } \\
\text { establishment of localization, } \\
\text { homeostatic process, protein } \\
\text { metabolic process, response } \\
\text { to stimulus, signalling, } \\
\text { system development }\end{array}$ & IL4 \\
\hline 10 & Glud1 & $\begin{array}{l}\text { glutamate } \\
\text { dehydrogenase } 1\end{array}$ & $\begin{array}{l}\text { establishment of } \\
\text { localization, response to } \\
\text { stimulus,signalling }\end{array}$ & TP53 \\
\hline
\end{tabular}

